\title{
Effects of hydrodynamic interaction on random adhesive loose packings of micron-sized particles
}

\author{
Wenwei Liu', Ran Tao ${ }^{1}$, Sheng Chen ${ }^{1}$, Huang Zhang ${ }^{1}$, and Shuiqing $\mathrm{Li}^{1, \star}$ \\ ${ }^{1}$ Key Laboratory for Thermal Science and Power Engineering of Ministry of Education, Department of Thermal Engineering, \\ Tsinghua University, Beijing 100084, China
}

\begin{abstract}
Random loose packings of monodisperse spherical micron-sized particles under a uniform flow field are investigated via an adhesive discrete-element method with the two-way coupling between the particles and the fluid. Characterized by a dimensionless adhesion parameter, the packing fraction follows the similar law to that without fluid, but results in larger values due to the hydrodynamic compression. The total pressure drop through the packed bed shows a critical behaviour at the packing fraction of $\phi \approx 0.22$ in the present study. The normalized permeability of the packed bed for different parameters increases with the increase of porosities and is also in consistent with the Kozeny-Carman equation.
\end{abstract}

\section{Introduction}

Understanding the properties of random packings of uniform spherical particles has drawing a lot of scientific attractions, since random packings are classical models of simple liquids, colloidal systems as well as granular matter [1-3] and also occur in a variety of engineering applications including fibrous filtration, industrial manufacturing, agriculture and physics $[4,5]$. In packings of large grains where elastic and frictional forces dominate, the packing fraction $\phi$ expands between two well-known limits, the random close packing $(\mathrm{RCP})$ limit at $\phi_{\mathrm{RCP}} \approx 0.64$ and the random loose packing (RLP) limit at $\phi_{\mathrm{RLP}} \approx 0.55$ $[1,2,6,7]$. However, most of the real particles in the nature are micron-sized, which are subject to not only elastic and frictional forces, but also adhesive forces, electrostatic forces and hydrodynamic forces $[4,5]$. In this case, both the structural and mechanical properties of microparticle packings could be intrinsically different in presence of such interactions.

Previous simulations have found that both the volume fraction and the coordination number of micro-particle packings can go far below the RLP limit when considering the van der Waals adhesive forces $[8,9]$. The effect of adhesion can be characterized by a dimensionless adhesion parameter, which uniquely determines the packing fraction $\phi$ and coordination number $Z$ [8-10] with fixed friction coefficient. The introduction of electrostatic repulsion reduces both $\phi$ and $Z$ due to the deceleration of the incoming particles, resulting in weaker inertial impaction and looser packing structure [11]. As for experimental investigations, a relatively low packing fraction of $\phi=0.15$ was achieved by random ballistic deposition of $1.5 \mu \mathrm{m}$ particles in vacuum [12]. Similar results were obtained with

\footnotetext{
^e-mail: lishuiqing@tsinghua.edu.cn
}

fluidization technique that jamming of $7.8-19.1 \mu \mathrm{m}$ particles takes place in the range $\phi=0.23-0.33$, which is related to the attractive energy [13]. Beyond jamming, the increase of hydrodynamic pressure results in the compression and densification of the micro-particle packing, and the applied stress is found to follow a power-law scaling $\sigma \sim\left(\phi-\phi_{J}\right)^{\beta}$ near jamming.

In this work, we study the random packings of micronsized spherical particles under a uniform flow field by means of a CFD-DEM coupled approach. The influence of the particle clusters on the fluid flow is well considered, through a two-way coupling of the fluid motion and the particle motion. The packing fraction with different material properties is examined and regressed with respect to the adhesion parameter. The pressure drop through the packing as well as the permeability are also analysed and discussed.

\section{Models and method}

\subsection{Two-way coupled CFD-DEM approach}

We perform numerical simulations of micro-particle packings via a discrete element method (DEM), which is coupled into an framework of the open-source CFD codes MFIX. The governing equations of the fluid-phase involve the mass and momentum conservation, which can be expressed as

$$
\begin{aligned}
& \frac{\partial \epsilon_{\mathrm{f}}}{\partial t}+\nabla \cdot\left(\epsilon_{\mathrm{f}} \mathbf{u}\right)=0, \\
& \frac{D}{D t}\left(\epsilon_{\mathrm{f}} \rho_{\mathrm{f}} \mathbf{u}\right)=\nabla \cdot \bar{S}_{\mathrm{f}}-\mathbf{I}_{\mathrm{fs}} .
\end{aligned}
$$

Here, $\epsilon_{\mathrm{f}}$ is the fluid-phase volume fraction, $\rho_{\mathrm{f}}$ is the mass density of the fluid, $\mathbf{u}$ is the volume-averaged fluid velocity, $\bar{S}_{\mathrm{f}}$ is the stress tensor of the fluid and $\mathbf{I}_{\mathrm{fs}}$ is the momentum transfer term between the fluid and particle phases. 
The computations of the flow field and the particles' information are two-way coupled through the the momentum transfer, which means that in every time step the flow field is first updated in the presence of the particle phase and then the forces acting on the particles are recalculated with the newest flow field. The DEM framework used in this work is specifically developed for micro-particles. The multi-coupling of adhesive force, friction and other interactions in the short-range particle-particle interaction zone is well tested and validated by particle/surface impact experiments $[4,5]$. Both the translational and rotational motions of each particle follow the Newton's second law,

$$
\begin{aligned}
m \frac{d \mathbf{v}}{d t} & =\mathbf{F}_{\mathrm{f}}+\mathbf{F}_{\mathrm{a}}, \\
I \frac{d \mathbf{\Omega}}{d t} & =\mathbf{M}_{\mathrm{f}}+\mathbf{M}_{\mathrm{a}},
\end{aligned}
$$

where $\mathbf{v}$ and $\boldsymbol{\Omega}$ are, respectively, velocity and rotation rate of an individual particle, $m$ is the particle mass, and $I$ is the moment of inertia. Gravity is not considered here. $\mathbf{F}_{\mathrm{f}}$ and $\mathbf{M}_{\mathrm{f}}$ are the fluid forces and torques exerting on a particle. $\mathbf{F}_{\mathrm{a}}$ and $\mathbf{M}_{\mathrm{a}}$ denote the van der Waals contact forces and torques acting on the particle. They include

$$
\begin{aligned}
& \mathbf{F}_{\mathrm{a}}=F_{\mathrm{n}} \mathbf{n}+F_{\mathrm{s}} \mathbf{t}_{\mathrm{s}}, \\
& \mathbf{M}_{\mathrm{a}}=r_{\mathrm{p}} F_{\mathrm{s}}\left(\mathbf{n} \times \mathbf{t}_{\mathrm{s}}\right)+M_{\mathrm{r}}\left(\mathbf{t}_{\mathrm{s}} \times \mathbf{n}\right)+M_{\mathrm{t}} \mathbf{n},
\end{aligned}
$$

where $F_{\mathrm{n}}$ is the normal force including adhesively elastic contact force $F_{\text {ne }}$ and damping force $F_{\text {nd }}, F_{\mathrm{s}}$ is the tangential force due to the sliding friction, $M_{\mathrm{r}}$ is the rolling resistance and $M_{\mathrm{t}}$ is the twisting resistance. $r_{\mathrm{p}}$ is the particle radius. $\mathbf{n}, \mathbf{t}_{\mathrm{s}}$ and $\mathbf{t}_{\mathrm{r}}$ are the normal, tangential and rolling direction unit vectors, respectively. The Johnson-KendallRoberts (JKR) model is employed to account for $F_{\text {ne }}$ between relatively compliant micro-particles [14]. The sliding, twisting and rolling friction terms in the presence of adhesion, are all approximated by a spring-dashpot-slider model with model parameters given in Ref. [15]. The critical values $F_{s, \text { crit }}, M_{t, \text { crit }}$ and $M_{r \text {,crit }}$ are given by

$$
\begin{aligned}
F_{s, \text { crit }} & =\mu_{\mathrm{f}}\left|F_{\mathrm{ne}}+2 F_{\mathrm{C}}\right|, \\
M_{t, \text { crit }} & =3 \pi a F_{s, \text { crit }} / 16, \\
M_{r, \text { crit }} & =-4 F_{\mathrm{C}}\left(a / a_{0}\right)^{3 / 2} \Theta_{\text {crit }} R,
\end{aligned}
$$

meaning that once these limits are reached, the particles start to slide, spin or roll and the resistances stay constant. Here $\Theta_{\text {crit }}$ is the critical angle for the relative rolling between two particles, $F_{\mathrm{C}}$ is the critical pull-off force expressed by the surface energy $\gamma: F_{\mathrm{C}}=3 \pi \gamma R, R$ is the effective radius between two contacting particles $(1 / R=$ $\left.1 / r_{\mathrm{p} 1}+1 / r_{\mathrm{p} 2}\right)$, and $a$ is the radius of the contact area with $a_{0}$ at equilibrium in the JKR model.

\subsection{Simulation conditions}

As shown in Fig. 1, the simulation domain sizes with $H \times L \times L$, where $L=20 r_{\mathrm{p}}$ and $H=10 L$. The domain width $L$ is selected as the characteristic length scale, and the dimensionless computational domain is set as $-5 \leq X^{*} \leq 5$, $-0.5 \leq Y^{*} \leq 0.5$ and $-0.5 \leq Z^{*} \leq 0.5$, with $\mathrm{x}$-axis set along the direction of height. Periodic boundary conditions are set along the $Y^{*}$ and $Z^{*}$ directions. Our simulation

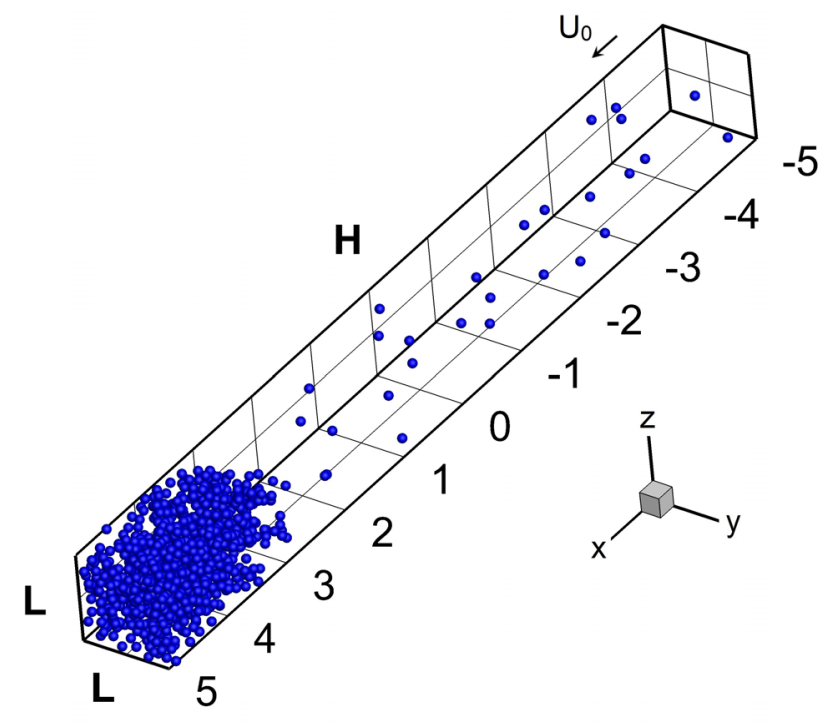

Figure 1. The schematic of the computational domain.

Table 1. Parameters used in the CFD-DEM simulations

\begin{tabular}{lll}
\hline Parameters & Value & Unit \\
\hline Particle & & \\
Particle radius $\left(r_{\mathrm{p}}\right)$ & 1 & $\mu \mathrm{m}$ \\
Mass density $\left(\rho_{\mathrm{p}}\right)$ & 2500 & $\mathrm{~kg} / \mathrm{m}^{3}$ \\
Elastic modulus $\left(E_{p}\right)$ & $2 \times 10^{7}$ & $\mathrm{~Pa}$ \\
Surface energy $(\gamma)$ & $0.03-125$ & $\mathrm{~mJ} / \mathrm{m}^{2}$ \\
Friction coefficient $\left(\mu_{\mathrm{f}}\right)$ & 0.3 & \\
Fluid (Air) & & \\
Mass density $\left(\rho_{\mathrm{f}}\right)$ & 1.25 & $\mathrm{~kg} / \mathrm{m}^{3}$ \\
Viscosity $(\mu)$ & $1.79 \times 10^{-5}$ & $\mathrm{~Pa} \cdot \mathrm{s}$ \\
Velocity $\left(U_{0}\right)$ & $0.01-5$ & $\mathrm{~m} / \mathrm{s}$ \\
\hline
\end{tabular}

starts from successive random injection of 2,000 uniform spheres at $X^{*}=-5$ with an initial velocity $U_{0}$. Note that the gravitational effect is eliminated, so that all the particles are transported by the fluid flow and deposit onto a plane at $X^{*}=5$. The initial flow field is set to be uniform, with $U_{x}=U_{0}, U_{y}=U_{z}=0$, and updated with the information of particles during every computational time step. Hence when the particles enter the domain, the flow field could be changed to be nonuniform. The physical parameters used in the simulations, including the particle and fluid properties, are all listed in Table 1. The friction coefficient is kept constant at $\mu_{\mathrm{f}}=0.3$. Two series of simulations were performed to investigate the effects of the physical parameters, where only one parameter was tuned while the others remained unchanged in each series. The tuned parameters are velocity and surface energy, respectively, of which the ranges can also be found in Table 1 .

\section{Results and discussions}

Figure 2 shows the packing fraction as a function of a dimensionless adhesion parameter $[8,11]$,

$$
\operatorname{Ad}=\frac{\gamma}{\rho_{\mathrm{p}} U_{0}^{2} R}
$$




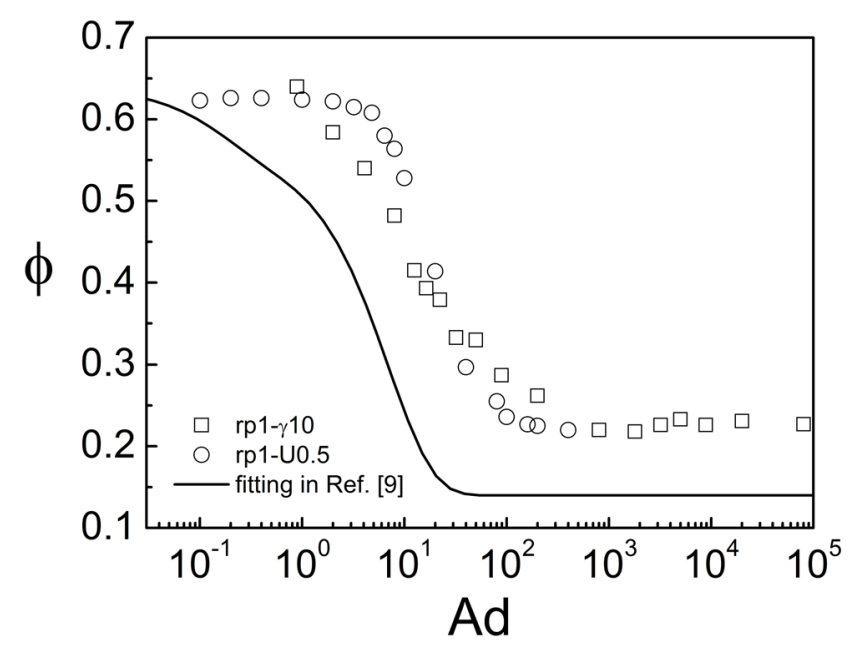

Figure 2. Packing fraction as a function of $A d$. "rp1- $\gamma 10$ " means that the particle radius and surface energy are fixed at $r_{\mathrm{p}}=1 \mu \mathrm{m}$ and $\gamma=10 \mathrm{~mJ} / \mathrm{m}^{2}$. The solid line is a fitting in Ref. [9] for microparticle packing in vacuum.

which represents the ratio of adhesion to particle inertia. It can be seen that with the increasing of $A d$, packing fraction decreases exponentially from close to $\phi_{\mathrm{RCP}}$ to a lower limit. A good regression can be observed for the two series simulations, as they still seem to fall on a single curve. However, the regression is not in consistent with that reported in Refs. [9] (the solid line), showing a shift to larger values of $A d$. Note that the packings in [9] were generated in a vacuum environment. The introduction of the fluid flow exerts continuous compression on the packed aggregates and results in denser structure, which gives rise to a larger packing fraction at the same $A d$. Since the flow velocity was fixed in each simulation case, a stable packing state will always come into being eventually due to the balance between fluid force and interparticle adhesive forces. The lowest packing fraction obtained in our simulations is around 0.22, which is higher than the conjectured ALP limit [8] due to the fluid compression. The highest packing fraction achieved is still around $\phi_{\mathrm{RCP}}$. This is because at this packing fraction, particles jam and geometrical constrains prevent the packing from reaching higher density through fluid compression.

In the study of the mechanical properties of the particle assembly, the consolidation stress plays an important role. Meanwhile, the pressure drop through packed bed is also an important parameter, since it serves as a fundamental property in fibrous filtration. Considering that gravity is not taken into account in our system, the normal consolidation stress applied on the packings equals the fluid pressure drop through the packed bed. It is well known that the pressure drop $\Delta P$ through a porous packed bed can be predicted by the Ergun function, or namely the KozenyCarman equation for a laminar flow field,

$$
\frac{-\Delta P}{h}=\frac{180 \mu U(1-\epsilon)^{2}}{d_{\mathrm{p}}^{2} \epsilon^{3}} .
$$

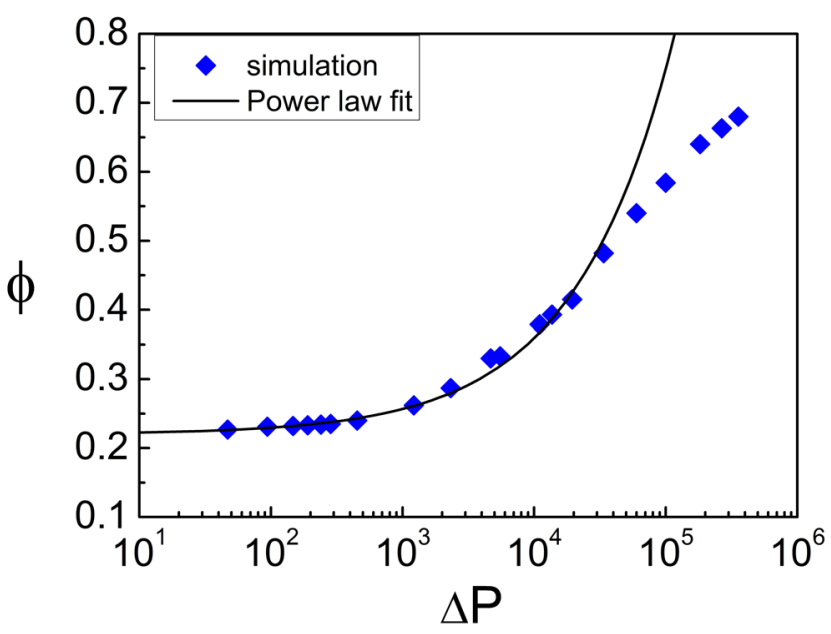

Figure 3. Variation of the applied stress with packing fraction. The solid line is a power-law fitting.

Here $h$ is the height of the packed bed, $U$ is the superficial fluid velocity and $\epsilon=1-\phi$ is the porosity of the packed bed. Figure 3 shows the variation of packing fraction with pressure drop. We see that the pressure drop derived from the simulations agrees well with the KozenyCarman equation, which testifies the validity of our numerical approach. Furthermore, when the fluid velocity is further decreased there comes a critical point at which the pressure drop gradually vanishes but the packing fraction stays. At this stage the clusters start to develop a yield stress, or in other words they are jammed [13]. It is also reported that the jamming point $\left(\phi_{\mathrm{J}}\right)$ for adhesive particles depends on the attractive energy, i.e. the surface energy in our study, instead of the particle size [13]. Here the jamming point found in the present work is $\phi_{\mathrm{J}} \approx 0.22$ for $\gamma=10 \mathrm{~mJ} / \mathrm{m}^{2}$. More data is needed to further explore the relationship between $\phi_{\mathrm{J}}$ and $\gamma$. Moreover, the stress near jamming is found to follow a power-law $\Delta P \sim\left(\phi-\phi_{\mathrm{J}}\right)^{\beta}$ [13]. The power-law fitting is plotted as the solid line in Fig. 3. The critical exponent is $\beta=1.788$ in our work, which is higher than $\beta=1.5$ for Hertzian-type interactions [13].

Apart from the pressure drop, permeability is also critically important in industries, especially in the composite manufacturing processes. Permeability describes the ability of porous materials to allow for fluid to flow through. Based on the Darcy's law, permeability is defined as $K=U \mu h / \Delta P$. Combining the Kozeny-Carman equation (Eq. 6), permeability can also be calculated as $K=d_{\mathrm{p}}^{2} \epsilon^{3} /\left(180(1-\epsilon)^{2}\right)$. From this definition, we can see that permeability depends on the particle size and the porosity. Thus, we can define a normalized permeability as

$$
K^{*}=\frac{K}{d_{\mathrm{p}}^{2}}=\frac{\epsilon^{3}}{\left(180(1-\epsilon)^{2}\right)} .
$$

In this case, permeability is only a function of packing porosity. Figure 4 shows the normalized permeability for different series simulations along with the theoretical prediction of Eq. 7. Generally, we can see that the permeabil- 


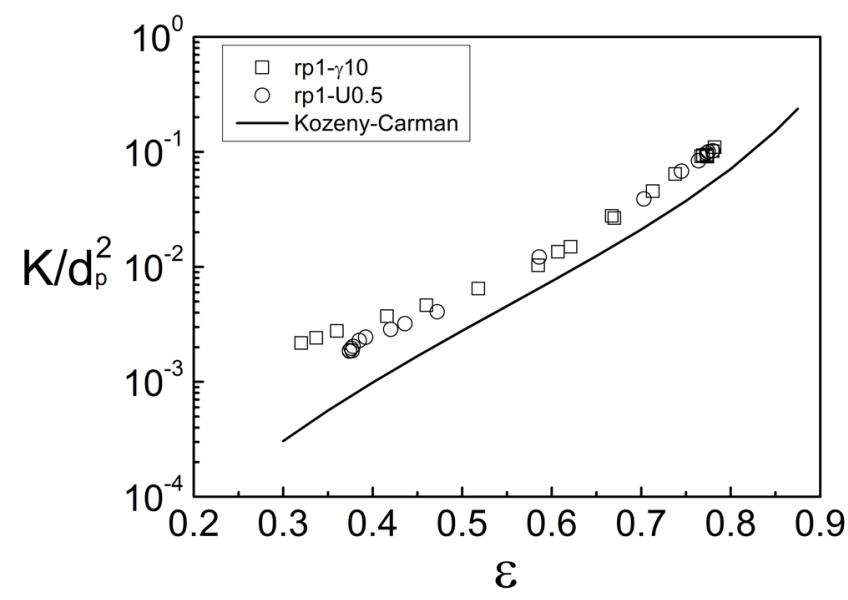

Figure 4. The normalized permeability as a function of the packing porosity. The solid line is the theoretical prediction derived from the Kozeny-Carman equation.

ity calculated from the simulation agrees with the theory, where a relatively large deviation occurs for small porosity.

\section{Conclusions}

In this work, a mesoscopic adhesive DEM incorporated with CFD approach was applied to investigate the random packings of monodisperse spherical micro-particles under a uniform flow field. The packing fraction with different material properties, the consolidation stress on the particle assembly and the permeability through the packed bed are examined and discussed. Conclusions are summarized as below.

(1) The packing fraction decreases with the increasing of a dimensionless adhesion parameter $A d$ but is higher than the reported value at the same $A d$, which is due to the hydrodynamic compression.

(2) The pressure drop, or the consolidation stress on the packed bed agrees well with the Kozeny-Carman equation and follows the power-law scaling $\Delta P \sim\left(\phi-\phi_{\mathrm{J}}\right)^{\beta}$ near the jamming point, which is found to be $\phi_{\mathrm{J}} \approx 0.22$ for $\gamma=10 \mathrm{~mJ} / \mathrm{m}^{2}$ under the present protocol.
(3) The normalized permeability agrees with the theoretical prediction derived from the Kozeny-Carman equation, except for a little deviation at low packing porosity.

\section{Acknowledgements}

This work has been funded by the National Natural Science Funds of China (Nos. 51390491) and the National Key Basic Research Program of China (No. 2013CB228506).

\section{References}

[1] J. D. Bernal, Nature 183, 141-147 (1959).

[2] G. Parisi, F. Zamponi, Rev. Mod. Phys. 82, 789-845 (2010).

[3] B. Andreotti, Y. Forterre, O. Pouliquen, Granular Media between fluid and solid (Cambridge University Press, Cambridge, 2013) p59-70.

[4] S. Q. Li, J. S. Marshall, G. Liu, Q. Yao, Prog. Energy Combust. Sci. 37, 633-668 (2011).

[5] J. S. Marshall and S.Q. Li, Adhesive Particle Flow: A Discrete-Element Approach (Cambridge University Press, Cambridge, 2014) p2,p81,p98.

[6] C. Song, P. Wang, H. A. Makse, Nature 453, 629-632 (2008).

[7] G. Y. Onoda, E. G. Liniger, Phys. Rev. Lett. 64, 2727 (1990).

[8] W. W. Liu, S. Q. Li, A. Baule, H. A. Makse, Soft Matter 11, 6492-6498 (2015).

[9] W. W. Liu, S. Q. Li, S. Chen, Powder Technol. 302, 414-422 (2016).

[10] S. Q. Li, J. S. Marshall, J. Aerosol Sci. 38, 1031-1046 (2007).

[11] S. Chen, S. Q. Li, W. W. Liu, H. A. Makse, Soft Matter 12, 1836-1846 (2016).

[12] J. Blum, R. Schräpler, Phys. Rev. Lett. 93, 115503 (2004).

[13] J. M. Valverde, M. A. S. Quintanilla, A. Castellanos, Phys. Rev. Lett. 92, 258303 (2004).

[14] K. L. Johnson, K. Kendall, A. D. Roberts, Proc. R. Soc. London A 324, 301-313 (1971).

[15] M. M. Yang, S. Q. Li, Q. Yao, Powder Technol. 248, 44-53 (2013). 